\title{
Risk factors for traumatic blunt cerebrovascular injury diagnosed by computed tomography angiography in the pediatric population: a retrospective cohort study
}

\author{
Vijay M. Ravindra, MD, ${ }^{1}$ Jay Riva-Cambrin, MD, MSc, ${ }^{1}$ Walavan Sivakumar, MD, ${ }^{1}$ \\ Ryan R. Metzger, PhD, ${ }^{2,3}$ and Robert J. Bollo, MD ${ }^{1}$
}

1Division of Pediatric Neurosurgery, Department of Neurosurgery, University of Utah School of Medicine; and 2Department of Surgery, University of Utah School of Medicine; ${ }^{3}$ Division of Pediatric Surgery, Primary Children's Hospital, Salt Lake City, Utah

OBJECT Computed tomography angiography (CTA) is frequently used to examine patients for blunt cerebrovascular injury (BCVI) after cranial trauma, but the pediatric population at risk for BCVI is poorly defined. Although CTA is effective for BCVI screening in adults, the increased lifetime risk for malignant tumors associated with this screening modality warrants efforts to reduce its use in children. The authors' objective was to evaluate the incidence of BCVI diagnosed by CTA in a pediatric patient cohort and to create a prediction model to identify children at high risk for BCVI.

METHODS Demographic, clinical, and radiographic data were collected retrospectively for pediatric patients who underwent CTA during examination for traumatic cranial injury from 2003 through 2013 . The primary outcome was injury to the carotid or vertebral artery diagnosed by CTA.

RESULTS The authors identified 234 patients (mean age 8.3 years, range $0.04-17$ years, 150 [64\%] boys) who underwent CTA screening for BCVI. Of these, $24(10.3 \%)$ had a focal neurological deficit, and $153(65.4 \%)$ had intracranial hemorrhage on a head CTA. Thirty-seven BCVls were observed in 36 patients (15.4\%), and 16 patients (6.8\%) died. Multivariate regression analysis identified fracture through the carotid canal, petrous temporal bone fracture, Glasgow Coma Scale (GCS) score of < 8, focal neurological deficit, and stroke on initial CT scan as independent risk factors for BCVI. A prediction model for identifying children at high risk for BCVI was created. A score of $\leq 2$ yielded a $7.9 \%$ probability of BCVI and a score of $\geq 3$ a risk of $39.3 \%$ for BCVI.

CONCLUSIONS For cranial trauma in children, fracture of the petrous temporal bone or through the carotid canal, focal neurological deficit, stroke, and a GCS score of < 8 are independent risk factors for BCVI.

http://thejns.org/doi/abs/10.3171/2014.11.PEDS14397

KEY WORDS cerebrovascular injury; computed tomography angiography; pediatrics; traumatic brain injury; trauma; technique

$\mathrm{B}$ LUNT cerebrovascular injury (BCVI) occurs in $1 \%$ of all blunt traumatic injuries in adults ${ }^{21}$ and in $3 \%-$ $4 \%$ of patients with identified risk factors. . $^{4,20,21}$ These injuries may lead to ischemia and significant neurological deficits, especially in the absence of early recognition and treatment. ${ }^{8,12}$ Computed tomography angiography (CTA) of the head and neck has emerged as the principal modality to screen patients for BCVI during the initial trauma evaluation. . $^{2,7,9,11,28}$
Risk factors for BCVI are well established in the adult trauma population, ${ }^{4,7,21}$ and studies have shown that BCVI screening in high-risk adult trauma patients may be cost effective. ${ }^{13}$ The Denver and Memphis criteria established a Glasgow Coma Scale (GCS) score of $<6$, basilar skull fracture, diffuse axonal injury, Le Fort Type II or III facial fractures, Horner syndrome, neurological examination results incompatible with brain imaging findings, cervical spine fracture, and neck hematoma as risk factors for

ABBREVIATIONS BCVI = blunt cerebrovascular injury; CTA = computed tomography angiography; EAST = Eastern Association for the Surgery of Trauma; GCS = Glasgow Coma Scale; ICA = internal carotid artery; NPTR = National Pediatric Trauma Registry; ROC = receiver operating characteristic; TBI = traumatic brain injury; VA = vertebral artery.

SUBMITTED July 28, 2014. ACCEPTED November 7, 2014.

INCLUDE WHEN CITING Published online March 6, 2015; DOI: 10.3171/2014.11.PEDS14397.

DISCLOSURE The authors report no conflict of interest concerning the materials or methods used in this study or the findings specified in this paper. 
BCVI.,21 Previous studies have suggested that BCVI complicates $0.03 \%-0.9 \%$ of traumatic brain injuries (TBIs) in children $^{1,12,14,17}$ and that the risk factors in this population may be similar to those seen in adults, ${ }^{14}$ however, recent evidence indicates that patterns of intracranial injury observed on head CT scans in children are significantly different from the patterns observed in adults..$^{24}$ This relatively low incidence has led to the speculation that children with TBI are inadequately screened, ${ }^{1}$ potentially increasing the number of children who undergo CTA.

Exposing children to radiation from $\mathrm{CT}$ has been directly linked to an increased risk for the development of future neoplasms, including leukemia and brain cancer. ${ }^{6,20,22}$ Current estimates suggest that 4.25 million CT scans are acquired in children in the United States annually, resulting in an estimated 4870 future cancers directly attributable to $\mathrm{CT} .{ }^{20} \mathrm{~A}$ retrospective cohort series found a 2.82-fold increased relative risk for brain cancer among children who underwent high-dose CT.22 It has been estimated that as many as 33\% of CT scans in children are unnecessary. ${ }^{6,10,26}$ Head CT is the most frequent type of CT imaging performed in children, and trauma is among the most common indications for it. ${ }^{20}$

Increasing awareness of the risks of radiation exposure from CT for children has led to the As Low As Reasonably Achievable (ALARA) concept, ${ }^{26}$ as well as to discussion in the lay press. ${ }^{23}$ The implementation of evidencebased clinical prediction rules in pediatric head injury has decreased unnecessary head CT scans in children at low risk for clinically significant head injury. ${ }^{15,25} \mathrm{We}$ sought to define independent risk factors for BCVI specific to the pediatric population and to develop a clinical prediction rule for identifying and screening high-risk pediatric patients while decreasing potentially unnecessary CT scanning in low-risk patients. ${ }^{6,10,26}$

\section{Methods \\ Patient Population}

We performed a retrospective review of the records of all patients who underwent CTA of the head or neck for suspected BCVI at a single pediatric Level I trauma center during an 11-year period (January 2003-December 2013). Before data collection, approval for this study was obtained from the institutional review and privacy boards.

\section{Data Collection}

The trauma registry at our hospital was queried to identify all patients who had a TBI during the period surveyed. The criteria for inclusion in the registry were at least one ICD-9-CM injury diagnostic code (800-849 for fractures, dislocations, and sprain of joints and adjacent muscles; 850-854 for intracranial injury without skull fracture; 860-869 for internal injury to chest, abdomen, or pelvis; 870-897 for open wounds of head, neck, trunk, or limbs; 900-904.99 for injury to blood vessels; $925-929.99$ for crush injuries; and 940-959.9 for burns, injuries to nerves and spinal cord, and traumatic complications and unspecified injuries). In addition, patients had to meet at least one of the following criteria: hospital admission, patient transfer between hospitals via emergency medical services, death resulting from traumatic injury independent of hospital admission or transfer status, or patient transport via air ambulance from any location. We recorded the total number of patients with TBI examined at our institution during the study period, and we also noted how many of these patients underwent a noncontrast CT of the head as part of their examination.

Next, we queried a radiological database to identify those patients who underwent screening for BCVI via CTA of the head or neck. The CTA was ordered at the discretion of the treating physician. We reviewed all relevant medical records and radiological reports and recorded demographics such as patient age, sex, and race, as well as clinical data, including injury type (penetrating injury, defined as any penetrating component, or blunt-force injury), injury event (motor vehicle accident, pedestrian struck, fall $>1$ story, nonaccidental trauma, or other), initial GCS score, presence of focal neurological deficits on initial examination, mode of treatment for TBI (medical or surgical), and associated fractures. Penetrating injury was included to determine whether a penetrating component was an independent risk factor in the event of a combination of penetrating and blunt traumas.

Radiological variables recorded included the year of the CTA, institution where the CTA was performed, presence of a fracture in the petrous temporal bone, presence of a fracture through the carotid canal, fracture type (linear or comminuted), presence of injury to the internal carotid artery (ICA) or vertebral artery (VA), type of brain injury (that is, epidural, subdural, or subarachnoid hemorrhage), presence of hypodensity on images consistent with ischemia, Rotterdam score (a 6-point score, based on findings on initial noncontrast $\mathrm{CT}$, that predicts the mortality rate at 6 months postinjury in moderate and severe TBI and has been validated in the pediatric population), ${ }^{18,19}$ and radiation dose delivered to the patient measured in dose-length product. The primary outcome was ICA or VA injury identified by CTA, classified according to the BCVI scale: ${ }^{3}$ a Grade 1 injury is characterized by intimal irregularity with $<25 \%$ narrowing, Grade 2 injury is a dissection or presence of intramural hematoma with $>25 \%$ narrowing, Grade 3 injury is the presence of a pseudoaneurysm, Grade 4 injury is an occlusion, and Grade 5 injury is transection with extravasation. ${ }^{3}$

\section{Statistical Analysis}

Data were summarized as the mean \pm SD for continuous variables and count and frequency for categorical variables. Univariate statistical analysis was performed to identify the unadjusted association of CTA-diagnosed BCVI with basic demographics and with clinical and radiographic risk factors. Between-group comparisons were analyzed with the Fisher exact test and with the chi-square test for categorical variables and with the Student t-test for continuous variables. Colinearity was determined with Pearson correlation coefficients.

Multivariate logistic regression analysis was performed with 2 categories of variables: 1) those satisfying statistical entry criteria $(\mathrm{p}<0.2)$ in the univariate analysis and 2) independent risk factors for traumatic BCVI previously identified in large adult studies, regardless of univariate 
TABLE 1. Basic demographic information and results of the univariate analysis of risk factors for patients who underwent CTA screening for BCVI*

\begin{tabular}{|c|c|c|c|c|}
\hline Variable & All Patients & Patients w/ BCVI & Patients w/o BCVI & $p$ Value \\
\hline \multicolumn{5}{|l|}{ Patient demographics } \\
\hline No. of patients & $234(100)$ & $36(15.4)$ & $198(84.6)$ & NA \\
\hline Mean age in yrs $\pm S D$ & $8.3 \pm 4.9$ & $8.5 \pm 5.1$ & $8.3 \pm 4.9$ & 0.77 \\
\hline Male sex & $150(64.1)$ & $23(63.9)$ & $127(64.1)$ & 0.98 \\
\hline White race & $163(69.7)$ & $24(66.7)$ & $138(69.7)$ & 0.70 \\
\hline Death & $16(6.8)$ & $5(13.9)$ & $11(5.6)$ & 0.08 \\
\hline \multicolumn{5}{|l|}{ Injury factors } \\
\hline Injury type & & & & 0.50 \\
\hline Penetrating & $11(4.7)$ & $2(5.6)$ & $9(4.6)$ & NA \\
\hline Blunt, high energy & $181(77.4)$ & $30(83.3)$ & $151(76.3)$ & NA \\
\hline Blunt, low energy & $42(18.0)$ & $4(11.1)$ & 38 (19.2) & NA \\
\hline Injury event & & & & 0.33 \\
\hline Motor vehicle accident & $60(25.6)$ & $11(30.6)$ & $49(24.8)$ & NA \\
\hline Struck pedestrian & $37(15.8)$ & $8(22.2)$ & $29(14.6)$ & NA \\
\hline Fall $>1$ story & $18(7.7)$ & $0(0.0)$ & $18(9.1)$ & NA \\
\hline Nonaccidental trauma & $4(1.7)$ & $0(0.0)$ & $4(2.0)$ & NA \\
\hline Ground-level fall & $42(18.0)$ & $4(11.1)$ & 38 (19.2) & NA \\
\hline Penetrating & $11(4.7)$ & $2(5.6)$ & $9(4.6)$ & NA \\
\hline Hanging & $18(7.7)$ & $0(0.0)$ & $18(9.1)$ & NA \\
\hline Other & $62(26.5)$ & $11(30.6)$ & $51(25.8)$ & NA \\
\hline \multicolumn{5}{|l|}{ Clinical examination } \\
\hline GCS score $\leq 8$ & $108(46.2)$ & $26(72.2)$ & $82(41.4)$ & $<0.001$ \\
\hline Focal neurological deficit & $24(10.3)$ & $10(27.8)$ & $14(7.1)$ & $<0.001$ \\
\hline \multicolumn{5}{|l|}{ Imaging } \\
\hline Rotterdam score $>3$ & $10(4.3)$ & $4(11.1)$ & $6(3.0)$ & 0.05 \\
\hline Any hemorrhage on $\mathrm{CT}$ & $153(65.4)$ & $27(75.0)$ & $126(63.6)$ & 0.25 \\
\hline Cerebral infarct on CT & $14(6.0)$ & $6(16.7)$ & $8(4.0)$ & 0.01 \\
\hline Epidural hematoma & $31(13.3)$ & $5(13.9)$ & $26(13.1)$ & 0.90 \\
\hline Subdural hematoma & $65(27.8)$ & $11(30.6)$ & $54(27.3)$ & 0.69 \\
\hline Traumatic SAH & $54(23.1)$ & $11(30.6)$ & $43(21.7)$ & 0.28 \\
\hline Contusion & $66(28.2)$ & $14(38.9)$ & $52(26.3)$ & 0.16 \\
\hline \multicolumn{5}{|l|}{ Associated fractures } \\
\hline Petrous temporal bone fracture & $18(7.7)$ & $5(13.9)$ & $13(6.6)$ & 0.17 \\
\hline Fracture through carotid canal & $60(25.6)$ & $16(44.4)$ & $44(22.2)$ & 0.007 \\
\hline Other skull base fracture & $37(15.8)$ & $6(16.7)$ & $31(15.7)$ & 0.80 \\
\hline Associated spine fracture & $18(7.7)$ & $2(5.6)$ & $16(8.1)$ & 0.60 \\
\hline \multicolumn{5}{|l|}{ Surgical management } \\
\hline Any surgical management & $65(27.8)$ & $14(38.9)$ & $51(25.8)$ & 0.11 \\
\hline ICP monitoring & $31(13.2)$ & $7(19.4)$ & $24(12.1)$ & 0.29 \\
\hline External ventricular drain & $36(15.4)$ & $10(27.8)$ & $26(13.1)$ & 0.08 \\
\hline Craniotomy & $32(13.7)$ & $7(19.4)$ & $25(12.6)$ & 0.30 \\
\hline
\end{tabular}

statistical association in our data set.,21 Calibration was examined using the Hosmer-Lemeshow goodness-of-fit test, and the accuracy of the multivariate model was determined by using the receiver operating characteristic (ROC) c-statistic. ${ }^{16}$ Statistical significance was established at $\mathrm{p}<0.05$. We created a prediction model by converting odds ratios into integers while maintaining their individual numerical relationships. ${ }^{27}$ The prediction score was dichotomized into high- and low-risk strata, using the technique described by Weinstein and Fineberg. ${ }^{29}$ False- 


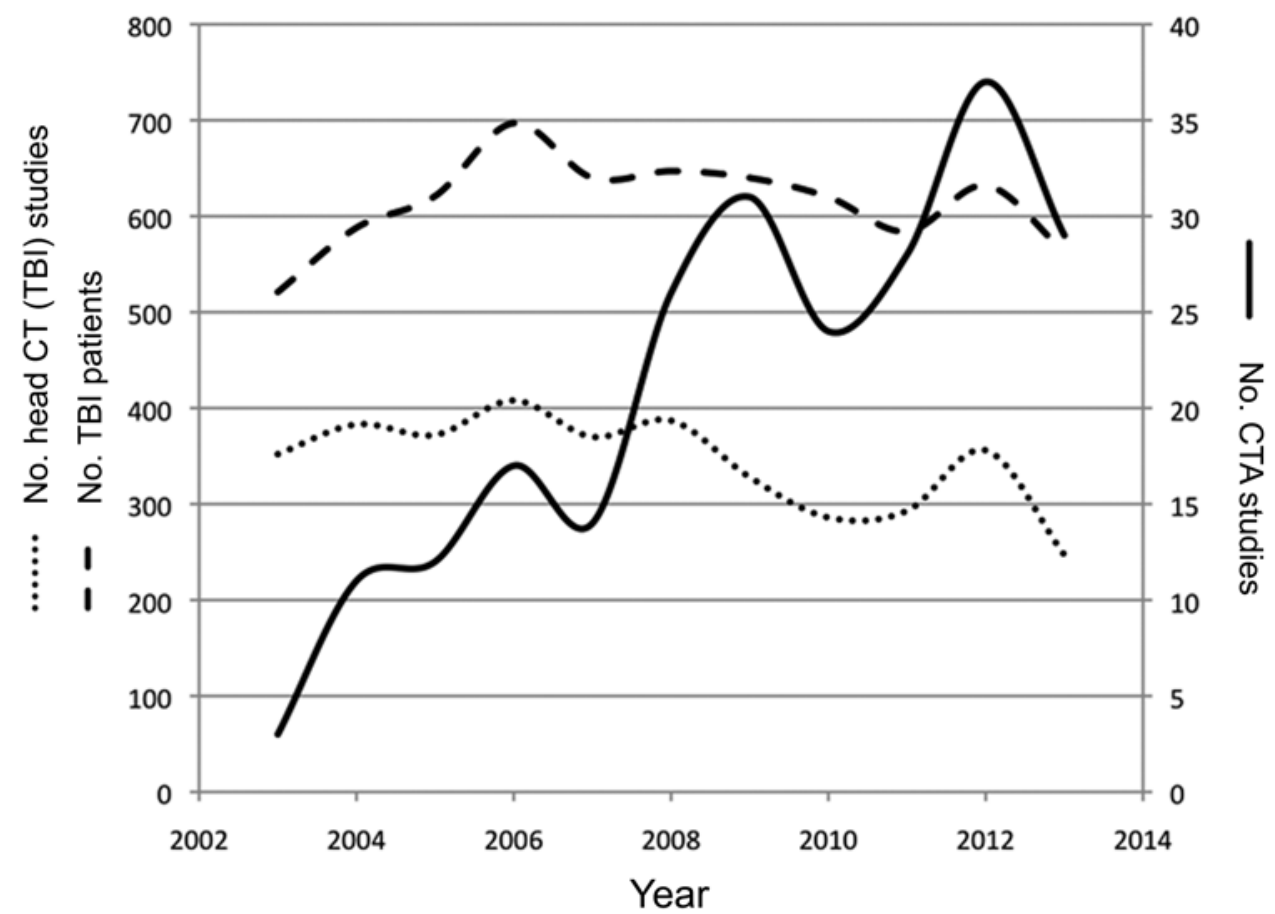

FIG. 1. Line graph showing changes in the number of patients with TBI and of CT examinations performed during the period surveyed. While the number of patients evaluated (dashed line) and the number of head CT scans obtained (dotted line) at our institution were largely stable over time, the number of CTA studies increased dramatically during this period (solid black line).

positive and false-negative misclassifications were analyzed using the same method. Data were analyzed using SAS (version 9.2) software.

\section{Results}

\section{Incidence and Diagnosis of BCVI}

During the 11-year period surveyed, 6753 pediatric patients were examined for TBI, and 3782 TBI patients underwent a head CT. Among these patients, we identified 234 (mean age 8.3 years, range $0.04-17$ years, 150 boys [64\%] who underwent CTA screening for a BCVI (Table
1); these patients represented $3.5 \%$ of all patients examined for trauma and $6.2 \%$ of all TBI patients who underwent a noncontrast head CT during this period. Figure 1 shows that while the number of TBI patients and head CT scans remained relatively stable over time, the frequency of CTA examinations increased during that period.

The mean radiation dose-length product in the CTA images was $649 \pm 642.5 \mathrm{mGy}-\mathrm{cm}$. Figure 2 shows the indications for a CTA; the most common indication was fracture through the carotid canal $(23.5 \%)$. Twenty-four patients $(10.3 \%)$ had a focal neurological deficit, and 153 (65.4\%) had an intracranial hemorrhage on a head CT (Ta-

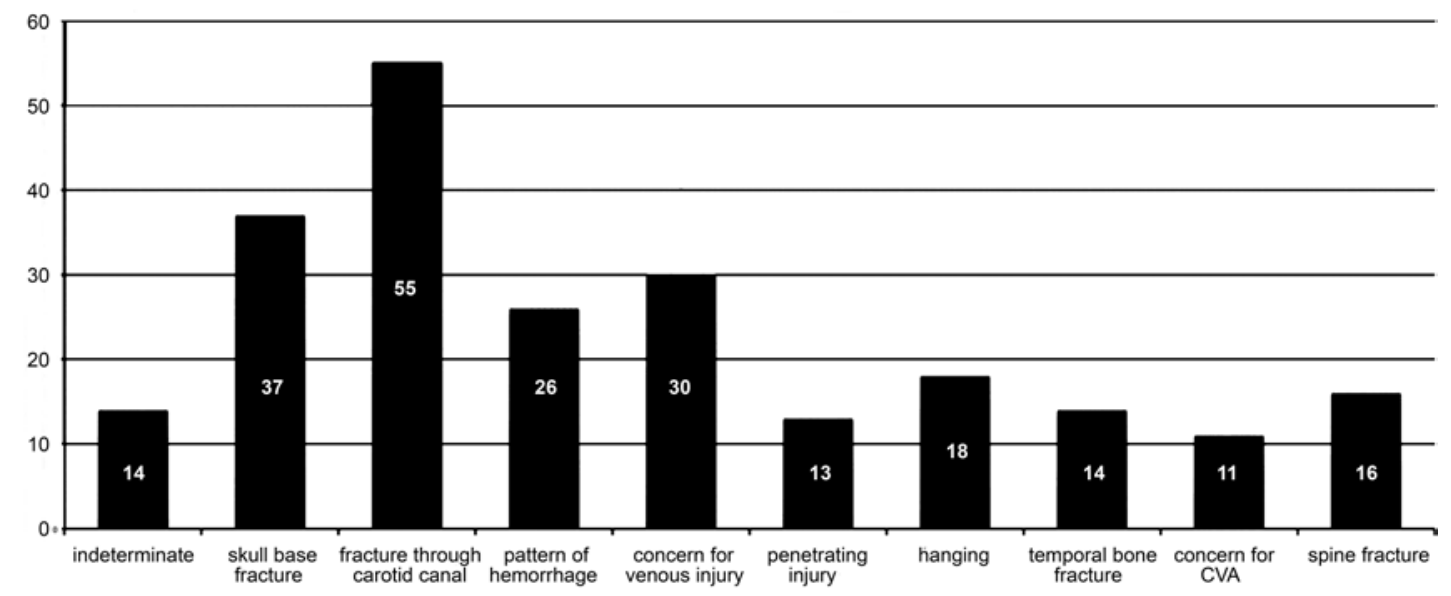

FIG. 2. Bar graph showing the various indications for CTA examination and the number of patients for each indication. CVA = cerebrovascular accident. 


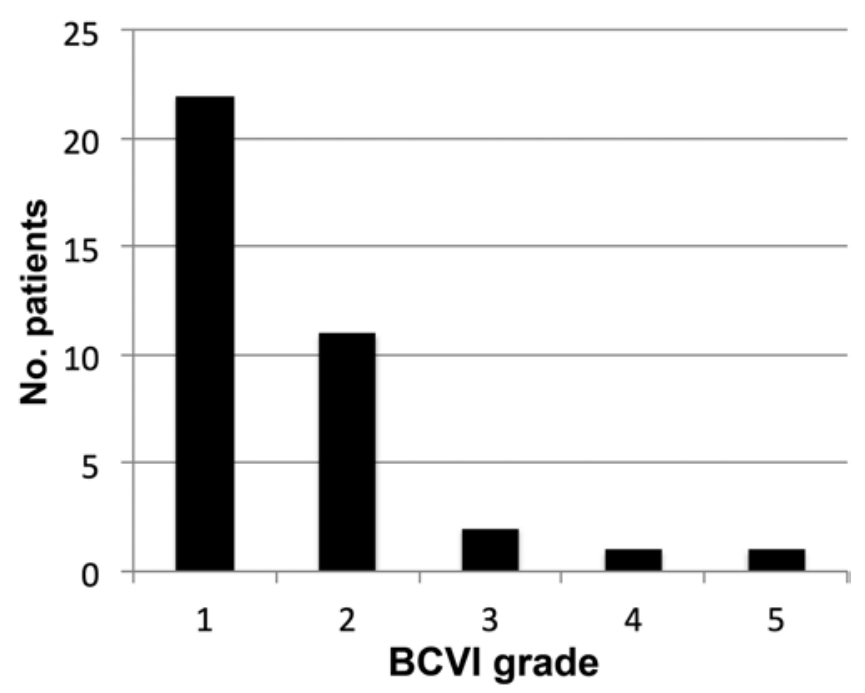

FIG. 3. Bar graph showing the distribution of BCVI grades of the injuries among the patients in this series. In total, 36 children had $37 \mathrm{BCVIs}$, of which 33 were injuries of the ICA and 4 of the VA.

ble 1). Thirty-seven BCVIs were identified in 36 patients, representing $15.4 \%$ of patients who underwent a CTA, $1 \%$ of patients with TBI who underwent a head CT, and $0.5 \%$ of all patients with TBI. Of these 36 patients with BCVI, $33(91.7 \%)$ had an ICA injury and $4(11.1 \%)$ had a VA injury (1 patient had both); 22 (59.5\%) of the BCVIs were Grade 1, 11 (29.7\%) were Grade 2, 2 (5.4\%) were Grade 3 , and $1(2.7 \%$ ) each were Grade 4 and Grade 5 (Fig. 3). Five of the 36 patients died in the hospital (representing $13.9 \%$ of patients with a BCVI identified by CTA) as did $11(5.6 \%)$ of 198 patients who underwent CTA without an identified BCVI. Only 9 (24.3\%) of the 37 BCVIs required management, consisting of antiplatelet therapy, anticoagulation, or an endovascular or open surgical procedure. All decisions to treat and on the mode of treatment were made on a case-by-case basis and involved a multidisciplinary team, including pediatric surgeons, pediatric neurosurgeons, pediatric neurologists, and endovascular surgeons.

Univariate analysis indicated that several clinical variables were significantly or almost significantly associated with a BCVI identified on CTA, including death $(\mathrm{p}=0.08)$, a GCS score of $\leq 8(p<0.001)$, and a focal neurological deficit on initial examination $(\mathrm{p}<0.001)$ (Table 1). No ICA or VA injuries were observed in patients who had hanging injuries $(\mathrm{p}=0.08)$. Among radiographic variables, a Rotterdam score of 4-6 $(\mathrm{p}=0.05)$, presence of a hypodensity on CT scans consistent with ischemia $(\mathrm{p}=0.01)$, and fracture through the carotid canal $(\mathrm{p}=0.007)$ were all significantly associated with BCVI. High-energy blunt trauma and some Memphis and Denver criteria, ${ }^{20,21}$ including temporal bone fracture, other skull base fractures, and spine fracture, were not significantly associated with BCVI but were included in a multivariate regression model because of their association with BCVI in adult trauma patients.

The variables and their statistical significance in multivariate regression analysis are shown in Table 2 . The independent risk factors statistically significantly associated with BCVI were fracture through the carotid canal, pe-
TABLE 2. Multivariate analysis of risk factors for patients who underwent CTA screening for BCVI*

\begin{tabular}{lcc}
\hline \multicolumn{1}{c}{ Variable } & $p$ Value & $\begin{array}{c}\text { Adjusted OR } \\
(95 \% \mathrm{Cl})\end{array}$ \\
\hline GCS score $\leq 8$ & 0.020 & $2.9(1.2-6.9)$ \\
\hline Focal neurological deficit & 0.004 & $4.6(1.6-13.0)$ \\
\hline Cerebral infarct on CT & 0.010 & $5.8(1.5-21.8)$ \\
\hline Fracture through the carotid canal & 0.002 & $4.3(1.7-10.8)$ \\
\hline Petrous temporal bone fracture & 0.004 & $6.8(1.9-24.6)$ \\
\hline * Risk factors that were also analyzed but were not statistically significant \\
$\begin{array}{l}\text { ( }>\text { > 0.05) included high-energy blunt injury, Rotterdam score > 3, skull base } \\
\text { fracture, spine fracture, and hanging. }\end{array}$
\end{tabular}

trous temporal bone fracture, GCS score of $\leq 8$, focal neurological deficit, and hypodensity consistent with ischemia on initial CT scan. The area under the ROC curve for the model was 0.81 , indicating a high degree of accuracy. The Hosmer-Lemeshow statistic was not significant, consistent with adequate model calibration.

\section{Development of a Clinical Prediction Score}

The results from this study cohort were used to create a prediction model (Table 3 ) and included the 5 variables identified as being independently associated with BCVI in our cohort (that is, a GCS of $\leq 8$, focal neurological deficit, ischemia on CT scan, fracture through the carotid canal, and petrous temporal bone fracture). According to the results of the multivariate logistic regression model, we developed the pediatric BCVI prediction score by rounding the odds ratios to the nearest integer while maintaining their relative numerical distance to one another. The newly developed score ranged from 0 (least likely to have BCVI) to 11 (most likely to have BCVI) (Table 4). The data dichotomized the cohort into a high-risk group and a low-risk group; the division into 2 groups may assist the clinician in the binary decision to screen a patient for BCVI during the initial trauma evaluation. The group of patients with a pediatric BCVI prediction score of $\leq$ 2 had a $7.9 \%$ probability of BCVI, whereas patients with a score of 3-11 had a $39.3 \%$ probability of BCVI. The pretest probability of BCVI identified by CTA screening

TABLE 3. Variables in the prediction model for BCVI and their assigned scores*

\begin{tabular}{lc}
\hline \multicolumn{1}{c}{ Variable } & Score \\
\hline GCS score $\leq 8$ & 1 \\
\hline Focal neurological deficit & 2 \\
\hline Carotid canal fracture & 2 \\
\hline Petrous temporal bone fracture & 3 \\
\hline Cerebral infarct on CT & 3 \\
\hline All & 11 \\
\hline
\end{tabular}

* Total possible scores range from 0-11. In our study cohort, a patient with a score $\leq 2$ had a $7.9 \%$ probability of $\mathrm{BCVI}$, and a patient with a score $\geq 3 \mathrm{had} \mathrm{a}$ $39.3 \%$ probability. 
TABLE 4. The pediatric BCVI prediction score

\begin{tabular}{ccc}
\hline Score & No. of Patients & Probability of BCVI* $(\%)$ \\
\hline 0 & 92 & 6.5 \\
\hline 1 & 56 & 10.7 \\
\hline 2 & 30 & 6.7 \\
\hline 3 & 37 & 32.4 \\
\hline 4 & 9 & 44.4 \\
\hline 5 & 7 & 57.1 \\
\hline 6 & 2 & 50.0 \\
\hline 7 & 0 & - \\
\hline 8 & 1 & 100.0 \\
\hline 9 & 0 & - \\
\hline 10 & 0 & - \\
\hline 11 & 0 & - \\
\hline
\end{tabular}

* The probability in the study population of having a BCVI with this score; dashes in this column denote missing data due to no patients with this score (the statistical significance of the model was $p<0.001$ ).

was 0.154 (15.4\%). For the low-risk group (comprising patients with a score of $\leq 2$ ), we observed a likelihood ratio of 0.47 , and using likelihood ratios, a posttest probability of $0.079(7.9 \%)$. Similarly, the likelihood ratio for the highrisk group was 3.59, and the posttest probability was 0.395 $(39.5 \%)$. Practitioners should have a high level of suspicion of BCVI in patients who have a hypodensity on CT scans suggesting infarction, a petrous temporal bone fracture, a carotid canal fracture coinciding with a GCS score of $\leq 8$, a focal neurological deficit coinciding with a GCS score of $\leq 8$, or a carotid canal fracture coinciding with a focal neurological deficit.

\section{Discussion}

In this study, we retrospectively identified 234 patients who underwent head or neck CTA after a traumatic injury. A BCVI was reported in 36 (15.4\%) of these patients. Multivariate regression analysis identified fracture through the carotid canal, petrous temporal bone fracture, GCS score of $\leq 8$, focal neurological deficit, and ischemia on noncontrast head CT as independent risk factors for BCVI. We created a prediction model that successfully identified children at high risk for BCVI.

\section{Pediatric BCVI}

Few previous studies have investigated BCVI in the pediatric trauma population.,12,14,17 Lew and colleagues ${ }^{17}$ reviewed the National Pediatric Trauma Registry (NPTR) over a 10-year period and identified 15 cases of blunt injury to the ICA among 57,659 individuals $<19$ years of age, but did not examine injuries to the VA. Two (13.3\%) of the 15 patients died. Skull base fracture, intracranial hemorrhage, chest trauma, clavicle fracture, and head and chest trauma were all significantly associated with blunt ICA injury. Because the NPTR relies on ICD-9 codes, the imaging modalities used to establish the diagnoses are unknown. ${ }^{17}$

More recently, Kopelman et al. ${ }^{14}$ retrospectively reviewed cases of BCVI among more than 1200 pediatric patients younger than 15 years examined for blunt traumatic injury at a Level I trauma center during a 5-year period. The authors analyzed risk factors according to the Eastern Association for the Surgery of Trauma (EAST) guidelines, ${ }^{7}$ incidence and method of evaluation, management, and outcome. Fewer than $40 \%$ of 127 patients who met the EAST criteria were screened with CTA during their initial examination; BCVI was identified in 11 (21\%) of the patients who were screened. ${ }^{14}$ Overall, BCVI was identified in $0.9 \%$ of the entire cohort. This observation is in contrast with those in our study, in which BCVI was identified in $15.4 \%$ of the patients with TBI who were screened with CTA. The observed differences in these variables between our study and that of Kopelman et al. ${ }^{14}$ are small and likely reflect variation between centers in the indications for screening CTA.

Kopelman and colleagues ${ }^{14}$ concluded that the risk factors for BCVI in children are similar to those in adults and recommended applying the EAST guidelines also to the pediatric trauma population. In contrast, after retrospectively reviewing findings on intracranial CT scans in 870 adults and 336 children examined after blunt trauma at a single center, Sarkar and colleagues ${ }^{24}$ reported that despite having similar GCS scores on admission, the 2 groups significantly differed in some findings. Specifically, the pediatric group was significantly more likely to present skull fracture and epidural hematoma and less likely to have a cortical contusion, subdural hemorrhage, or subarachnoid hemorrhage than the adult group. ${ }^{24}$

These observations may partly be explained by divergent mechanisms of injury; however, age-specific anatomical factors may also contribute to these differences between children and adults. These findings suggest that children and adults may also have different risk factors for BCVI. Jones et al. ${ }^{12}$ identified 45 children who had sustained blunt BCVIs and were examined after presenting at a single institution over a period of approximately 15 years. Although $72 \%$ of asymptomatic injuries met adult screening criteria, more than two-thirds of the patients who presented with acute neurological findings did not meet adult screening guidelines. The authors concluded that their data suggest that screening guidelines may need to be modified to account for the specific risk factors for BCVI in children. ${ }^{12}$

Lastly, in a large multicenter retrospective cohort study of 5829 children younger than 15 years who were examined for blunt traumatic injury, only $89(16.5 \%)$ of 538 patients who met adult criteria actually underwent screening for BCVI, and 23 BCVIs were identified in these patients. ${ }^{1}$ These data again highlight the importance of identifying specific BCVI risk factors and implementing a pediatricspecific scoring system.

In summary, we identified several independent risk factors for BCVI in a pediatric trauma cohort undergoing CTA screening for BCVI. Using these risk factors, we developed a clinical prediction rule to guide the use of CTA for evaluating pediatric patients at the highest risk for BCVI (Tables 3-4). While our results implicating petrous temporal bone fracture associated with an increased risk for BCVI in children are comparable with those from the adult population, ${ }^{4,21}$ we also determined that fracture 
through the carotid canal was an independent risk factor in children. Our data indicate that in children, a higher GCS score cutoff should be used than in adults and highlight the significance of focal neurological deficit and ischemia on noncontrast $\mathrm{CT}$, both of which are strongly and independently associated with BCVI. ${ }^{4}$ We did not identify patients with a BCVI attributable to spine fractures, but the external validity of this finding is limited by the small number of VA injuries in our data set.

\section{Limitations}

The limitations of this study are that we conducted a retrospective single-center study with inherent recall bias. We included only patients who underwent CTA imaging, representing only $6.2 \%$ of children with TBI who underwent a head CT. Therefore, we did not know how many patients with identified BCVI risk factors did not undergo CTA for examination. In total, $15.4 \%$ of screened patients had BCVI diagnosed on CTA scans. Patients with fractures of the petrous temporal bone and through the carotid canal were not included in our cohort if they did not undergo CTA imaging. This was especially likely during the first half of the period surveyed, when the frequency of screening was significantly lower than during the second half (Fig. 1). Further, with the exception of carotid canal fractures, we did not separately analyze different subtypes of petrous bone fracture, such as horizontal and longitudinal fractures. Le Fort II or III facial fractures, an independent risk factor for blunt BCVI in adults, ${ }^{4,21}$ were not analyzed in our data set and may be an important risk factor for BCVI in pediatric trauma as well. In addition, other possible risk factors including cervical soft-tissue injury and cervical pain or tenderness on examination were not included; the latter could not be assessed in the $46.2 \%$ of the study population who had GCS scores of $\leq 8$.

We developed a clinical prediction score to stratify patients to high-risk and low-risk groups. Patients with a score $\leq 2$ had a $7.9 \%$ risk of BCVI, whereas patients with a score $>2$ had a $39.3 \%$ risk of BCVI. A risk of $7.9 \%$ for a potentially lethal injury is still significant, and if there is strong clinical suspicion of BCVI despite a low prediction score, delayed MR angiography may be considered as an alternative screening modality that avoids radiation in lower-risk patients., ${ }^{5,28}$ Risk modeling in large adult populations is commonly used to guide BCVI screening in practice (with the Denver criteria) ${ }^{4}$ patients with no risk factors for blunt ICA injury and for whom screening is not recommended have a $20 \%$ risk for BCVI. By comparison, we have defined a pediatric population at much lower risk. We did not include patients with a vascular injury detected in a delayed fashion by another imaging modality in our data set, as it is not clear the injury would have been detected by CTA screening during the patient's initial trauma examination.

We identified only a small number of VA injuries in our series, so we cannot make definitive conclusions about the risk factors involved in these specific injuries. In our pediatric trauma registry during the period assessed, symptoms of TBI and cervical spine injury (fracture or ligamentous injury) were documented in 257 patients, of whom only $27(10.5 \%)$ underwent CTA. Because we could not retrospectively determine the incidence of vascular injury among patients who were not screened and whose injury may have been detected by the screening and because we did not evaluate ligamentous injury as a separate variable, our data set may have underestimated the association between spine injury and BCVI. Further, the findings of ICA or VA injury had varying clinical significance, in that only $24.3 \%$ of the BCVIs identified required management. Because our score has not been externally validated, we cannot recommend its widespread implementation; however, the risk factors used to create the score were strongly associated with BCVI in our trauma population and may be generally useful to identify children at high risk for BCVI who may benefit from CTA screening.

\section{Conclusions}

In a retrospective review of the records of 234 patients who underwent CTA screening after TBI at a single Level I pediatric trauma center in an 11-year period, we identified 5 risk factors for BCVI, including initial GCS score of $\leq 8$, fracture of the petrous temporal bone or through the carotid canal, ischemia on noncontrast head CT, and focal neurological deficit. On the basis of these data, we developed a clinical prediction rule to classify patients according to these risk factors as at low or high risk for BCVI. The prediction rule requires validation on an independent population and further prospective study.

\section{Acknowledgment}

We thank Kristin Kraus, MSc, for editorial assistance in preparing this paper.

\section{References}

1. Azarakhsh N, Grimes S, Notrica DM, Raines A, Garcia NM, Tuggle DW, et al: Blunt cerebrovascular injury in children: underreported or underrecognized?: A multicenter ATOMAC study. J Trauma Acute Care Surg 75:1006-1012, 2013

2. Biffl WL, Egglin T, Benedetto B, Gibbs F, Cioffi WG: Sixteen-slice computed tomographic angiography is a reliable noninvasive screening test for clinically significant blunt cerebrovascular injuries. J Trauma 60:745-752, 2006

3. Biffl WL, Moore EE, Offner PJ, Brega KE, Franciose RJ, Burch JM: Blunt carotid arterial injuries: implications of a new grading scale. J Trauma 47:845-853, 1999

4. Biffl WL, Moore EE, Offner PJ, Brega KE, Franciose RJ, Elliott JP, et al: Optimizing screening for blunt cerebrovascular injuries. Am J Surg 178:517-522, 1999

5. Bok AP, Peter JC: Carotid and vertebral artery occlusion after blunt cervical injury: the role of MR angiography in early diagnosis. J Trauma 40:968-972, 1996

6. Brenner DJ, Hall EJ: Computed tomography-an increasing source of radiation exposure. N Engl J Med 357:2277-2284, 2007

7. Bromberg WJ, Collier BC, Diebel LN, Dwyer KM, Holevar MR, Jacobs DG, et al: Blunt cerebrovascular injury practice management guidelines: the Eastern Association for the Surgery of Trauma. J Trauma 68:471-477, 2010

8. Carrillo EH, Osborne DL, Spain DA, Miller FB, Senler SO, Richardson JD: Blunt carotid artery injuries: difficulties with the diagnosis prior to neurologic event. J Trauma 46:11201125,1999

9. Chamoun RB, Mawad ME, Whitehead WE, Luerssen TG, Jea A: Extracranial traumatic carotid artery dissections in 
children: a review of current diagnosis and treatment options. J Neurosurg Pediatr 2:101-108, 2008

10. Donnelly LF: Reducing radiation dose associated with pediatric CT by decreasing unnecessary examinations. AJR Am J Roentgenol 184:655-657, 2005

11. Eastman AL, Chason DP, Perez CL, McAnulty AL, Minei JP: Computed tomographic angiography for the diagnosis of blunt cervical vascular injury: is it ready for primetime? J Trauma 60:925-929, 2006

12. Jones TS, Burlew CC, Kornblith LZ, Biffl WL, Partrick DA, Johnson JL, et al: Blunt cerebrovascular injuries in the child. Am J Surg 204:7-10, 2012

13. Kaye D, Brasel KJ, Neideen T, Weigelt JA: Screening for blunt cerebrovascular injuries is cost-effective. J Trauma 70:1051-1057, 2011

14. Kopelman TR, Berardoni NE, O'Neill PJ, Hedayati P, Vail SJ, Pieri PG, et al: Risk factors for blunt cerebrovascular injury in children: do they mimic those seen in adults? J Trauma 71:559-564, 2011

15. Kuppermann N, Holmes JF, Dayan PS, Hoyle JD Jr, Atabaki SM, Holubkov R, et al: Identification of children at very low risk of clinically-important brain injuries after head trauma: a prospective cohort study. Lancet 374:1160-1170, 2009

16. Lemeshow S, Hosmer DW Jr: A review of goodness of fit statistics for use in the development of logistic regression models. Am J Epidemiol 115:92-106, 1982

17. Lew SM, Frumiento C, Wald SL: Pediatric blunt carotid injury: a review of the National Pediatric Trauma Registry. Pediatr Neurosurg 30:239-244, 1999

18. Liesemer K, Riva-Cambrin J, Bennett KS, Bratton SL, Tran H, Metzger RR, et al: Use of Rotterdam CT scores for mortality risk stratification in children with traumatic brain injury. Pediatr Crit Care Med 15:554-562, 2014

19. Maas AI, Hukkelhoven CW, Marshall LF, Steyerberg EW: Prediction of outcome in traumatic brain injury with computed tomographic characteristics: a comparison between the computed tomographic classification and combinations of computed tomographic predictors. Neurosurgery 57:11731182,2005

20. Miglioretti DL, Johnson E, Williams A, Greenlee RT, Weinmann S, Solberg LI, et al: The use of computed tomography in pediatrics and the associated radiation exposure and estimated cancer risk. JAMA Pediatr 167:700-707, 2013

21. Miller PR, Fabian TC, Croce MA, Cagiannos C, Williams JS, Vang M, et al: Prospective screening for blunt cerebrovascular injuries: analysis of diagnostic modalities and outcomes. Ann Surg 236:386-395, 2002

22. Pearce MS, Salotti JA, Little MP, McHugh K, Lee C, Kim $\mathrm{KP}$, et al: Radiation exposure from CT scans in childhood and subsequent risk of leukaemia and brain tumours: a retrospective cohort study. Lancet 380:499-505, 2012
23. Redberg RF, Smith-Bindman R: We are giving ourselves cancer. New York Times. January 30, 2014. (http://nyti.ms/ MCbBTY) [Accessed January 22, 2015]

24. Sarkar K, Keachie K, Nguyen U, Muizelaar JP, ZwienenbergLee M, Shahlaie K: Computed tomography characteristics in pediatric versus adult traumatic brain injury. J Neurosurg Pediatr 13:307-314, 2014

25. Schonfeld D, Bressan S, Da Dalt L, Henien MN, Winnett JA, Nigrovic LE: Pediatric Emergency Care Applied Research Network head injury clinical prediction rules are reliable in practice. Arch Dis Child 99:427-431, 2014

26. Slovis TL: Children, computed tomography radiation dose, and the As Low As Reasonably Achievable (ALARA) concept. Pediatrics 112:971-972, 2003

27. Tu JV, Naylor CD: Clinical prediction rules. J Clin Epidemiol 50:743-744, 1997

28. Wang AC, Charters MA, Thawani JP, Than KD, Sullivan SE, Graziano GP: Evaluating the use and utility of noninvasive angiography in diagnosing traumatic blunt cerebrovascular injury. J Trauma Acute Care Surg 72:1601-1610, 2012

29. Weinstein M, Fineberg HV: The use of diagnostic information to revise probabilities, in Weinstein M, Fineberg HV, Elstein AS, et al (eds): Clinical Decision Analysis. Philadelphia: WB Saunders, 1980, pp 84-89

\section{Author Contributions}

Conception and design: Bollo, Riva-Cambrin. Acquisition of data: Ravindra, Sivakumar. Analysis and interpretation of data: Riva-Cambrin. Drafting the article: all authors. Critically revising the article: all authors. Reviewed submitted version of manuscript: all authors. Approved the final version of the manuscript on behalf of all authors: Bollo. Statistical analysis: Riva-Cambrin Administrative/technical/material support: Metzger. Study supervision: Bollo, Riva-Cambrin.

\section{Supplemental Information \\ Proceedings}

Preliminary versions of this work were presented at the 2014 Annual Meeting of the American Society of Pediatric Neurosurgeons (J Neurosurg Pediatr 13:A342-A354, 2014), the 2014 Annual Richard Lende Winter Neurosurgery Conference, and the Annual Meeting of the American Association of Neurological Surgeons, April 5-9, 2014, San Francisco, CA.

\section{Correspondence}

Robert J. Bollo, Division of Pediatric Neurosurgery, Primary Children's Hospital, 100 N. Mario Capecchi Dr., Ste. 1475, Salt Lake City, UT 84113. email: robert.bollo@hsc.utah.edu. 